\title{
ANALISIS CINEMÁTICO Y DINAMICO EN EL PATEO EN EL FUTBOL SALA
}

Sanabria, $Y^{1}$, Agudelo, $C^{2}$

1. Yofre Danilo Sanabria Arguello, Licenciado en Educación Física. Magister en Pedagogía de la Cultura Física. Docente de la Universidad Pedagógica y Tecnológica de Colombia. Correo: yofresanar6@gmail.com

2. Carlos Albero Agudelo Velásquez, Licenciado en Educación Física. Especialista en Entrenamiento Deportivo. Magíster en Motricidad. Docente de la Universidad de Antioquia.

\section{RESUMEN}

La biomecánica deportiva ofrece una variedad de técnicas para el análisis cinemático y dinámico de un gesto deportivo, lo cual permite medir con precisión el nivel técnico de una ejecución para este caso el de un pateo y sus posibles aspectos a perfeccionar, lo que podría ayudar a tener un mejor rendimiento deportivo del jugador de futbol sala, en este sentido la biomecánica es importante para analizar y con base en tal análisis potenciar las cualidades de cualquier movimiento. Según lo expuesto anteriormente la investigación tuvo como finalidad el estudio cinemático y dinámico del pateo en el futbol sala, para el cual se utilizó el programa kinovea, y una cámara desde un plano sagital, permitiendo realizar un análisis del gesto en ejecución.

El presente artículo incluye cuatro ítems. Primero la formulación del problema la cual hace referencia a la importancia de realizar un análisis cinemático y dinámico del pateo en el futbol sala. A continuación el marco teórico en donde se visualiza la temática específica del trabajo como es: Qué es la biomecánica, la biomecánica en el deporte, centros de gravedad, dinámica del movimiento, cinemática de movimiento y características del fútbol sala. Posteriormente encontramos la metodología que se empleó para el desarrollo del trabajo. Finalmente se presenta la interpretación y análisis de resultados resaltando lo que se puede lograr con las facilidades que proporcionan los pocos equipos utilizados.

Palabras clave: Biomecánica, futbol sala.

\section{KINEMATIC AND DYNAMIC ANALYSIS IN RELEASE IN FUTSAL}

\begin{abstract}
Sports biomechanics offers a variety of techniques for kinematic and dynamic analysis of a sporting gesture, allowing better accuracy discover the technical level of implementation during the kick and possible ways to improve, so we can have a better athletic performance futsal player in this regard biomechanics is important to analyze the qualities of any movement .

According to the above research was aimed at the study of the kinematic and dynamic kick in indoor soccer, for which the Kinovea program , and a camera was used from a sagittal plane, allowing for a analysis of gesture execution. To better understand the present work is divided into four items. First find the problem formulation which refers to the importance of a kinematic and
\end{abstract}


dynamic analysis of kicking in futsal Then we find as regards the theoretical framework where the specific theme of the work is displayed as: What is biomechanics, sports biomechanics, centers of gravity , movement dynamics, kinematics of motion and features football. Later we find the methodology used for development work. Finally, the interpretation and analysis of results using graphics programs and photo with comments is presented.

Keywords: biomechanics, indoor soccer .

\section{INTRODUCCIÓN}

¿Qué análisis es posible realizar de un pateo en fútbol sala utilizando sólo una cámara y el programa Kinovea?.

Se tienen pocos estudios prácticos sobre el análisis biomecánico en el gesto deportivo específico como es el pateo en el futbol sala probablemente porque se piensa que hacer biomecánica es costoso, esta investigación quiere mostrar con un ejemplo práctico que con pocos recursos y un conjunto de conocimientos aplicativos es posible realizar un análisis que conlleven a conclusiones particulares sobre la ejecución de un sujeto.

El fútbol sala cobra fuerza y cuenta cada vez con mayor número de seguidores y practicantes. Del mismo modo, el deporte en general y el fútbol sala en particular han alcanzado una situación en la cual prima la profesionalización de sus jugadores, de la misma manera, todo jugador necesita de un diagnóstico personal de capacidades en lo físico y en lo técnico, y una planificación de trabajos sistemáticos que se ajusten a esa evaluación, dando así solución práctica a uno de los aspectos más importantes en el tema de los principios del entrenamiento, concretamente al principio de individualización, ya que distintos autores se refieren a este principio como uno de los más fundamentales en el deporte de rendimiento. Para algunos autores es el principio básico de su propuesta de sistema de planificación (Agudelo, 2012).

Una de las formas de hacer explícito el principio de individualización es con el entrenamiento de la técnica, los entrenadores deben emplear métodos de entrenamiento individualizados enfocados en la enseñanza, desarrollo y perfeccionamiento de la técnica. Es por esto que se hace importante el desarrollo de investigaciones como está la cual permitirá a quienes trabajan en el medio, tener un ejemplo práctico de análisis de una técnica particular, lo que puede ser transferido a sus procesos de formación en esta modalidad 0 en otras, lo que puede ser un aporte en ese objetivo común de todos los entrenadores de lograr el mejoramiento $y$ perfeccionamiento del gesto particular de su respectiva modalidad deportiva. Para el caso del pateo en el futbol sala se presenta en esta investigación de caso un análisis que busca potenciar la forma de ser lo más efectivo posible lo que resulta vital para aquellos niveles de formación, rendimiento y competencia. Para los entrenadores, docentes de educación física y practicantes del deporte, es de vital importancia conocer elementos de carácter científico que posiblemente puedan incrementar el rendimiento para la consecución de mejores resultados en este deporte.

Es por tal razón que La investigación realizada pretende ser una herramienta que de ayuda para entrenadores, docente de educación física deportistas y quienes trabajan en el campo del deporte en general y del futbol sala en particular, brindándoles la posibilidad de conocer los elementos importantes que componen el pateo en el futbol sala y los componentes más relevantes de los cuales depende la 
efectividad del gesto, propiciando que se incorporen elementos al control de la preparación técnica lo que podrá abogar por una mejor preparación y por ende mejores resultados. El objetivo es analizar el fundamento del pateo en el futbol sala desde la cinemática y dinámica.

Fases del movimiento

Meinel y Shnabel(1988) proponen dividir el transcurso del gesto deportivo en partes 0 procesos parciales que suceden en el tiempo, concluyendo sobre este la división existente en secuencias ordenadas en el tiempo, en las que:

Fase preparatoria: se prepara el movimiento de la fase principal

Fase principal: se realiza y ejecuta el principal objetivo del gesto deportivo

Fase final: finaliza el gesto y se prepara el enlace con la acción siguiente

Biomecánica:

Según Hay (1978) la Biomecánica es la ciencia que examina las fuerzas actuando Sobre y en una estructura biológica y los efectos que producen dichas fuerzas.

Para Milburn, (1996) el concepto de Hay, implica un entendimiento del movimiento humano en tres áreas estructuras biológicas, análisis mecánicos y un entendimiento del movimiento. A través del saber de Anatomía, histología o mecánica, por si mismas no podrán avanzar hacia el entendimiento del movimiento humano. Es entonces, la habilidad de integrar multi disciplinariamente este conocimiento para proveer un entendimiento del movimiento lo que determina un correcto y profundo análisis del ambiente dinámico del movimiento.

Biomecánica del deporte:

La biomecánica deportiva, algunos autores refieren que se origina desde la cinesiología, por la razón de que las técnicas deportivas son adaptaciones de los movimientos básicos (patrones motores básicos) (Gutiérrez, 2007).

El objeto de estudio en esta disciplina de la biomecánica son las técnicas deportivas, las cuales se agrupan por deportes según sus similitudes (Izquierdo, 2008).

Las tareas de la biomecánica deportiva se dividen en tres áreas con sus respectivas tareas (Aguado, 1993):

Material Deportivo.

- Reducción de peso del material deportivo sin la pérdida de sus características.

- Ofrecer nuevos aparatos y metodologías de registros.

- Aumentar la durabilidad del material deportivo.

- Materiales que mejoren las marcas deportivas.

- Deportista.

- Describir las técnicas deportivas.

- Corregir defectos de la técnica deportiva.

- Proponer técnicas más eficientes y

eficaces.

- Medio Ambiente

- Minimizar las fuerzas de resistencia.

- Optimizar la propulsión en los diferentes medios.

La biomecánica deportiva se ocupa de describir y evaluar la técnica de los deportistas con el propósito de aumentar el rendimiento. Los estudios biomecánicos sobre las técnicas deportivas se orientan sobre los análisis cinemáticos: que estudian los gestos deportivos a través de métodos descriptivos sin tener en cuenta las causas que lo producen y los análisis cinéticos: estudian los gestos deportivos considerando las fuerzas que actúan sobre el movimiento y la mecánica muscular: que es el estudio de las fuerzas internas que produce el 
propio sistema en movimiento (Campos, 2003)

\section{Técnica}

Según la Real Federación Española de Fútbol Sala, técnica "es el gesto que ha de realizar el jugador, en cada una de las acciones que interviene en el desarrollo del juego". Esto abarca, tanto la defensa como el ataque, en posesión o no del balón.

Montero dice que son "todas las acciones 0 gestos que de forma precisa puede realizar un jugador con el balón".

\section{Análisis del movimiento}

Ramón (2006) afirma que el estudio del movimiento humano se debe realizar de dos formas: el análisis cuantitativo y el cualitativo. El análisis cuantitativo implica la descripción de los movimientos del cuerpo 0 sus partes, en términos numéricos. El observador puede entonces usar esta cuantificación para explicar o describir la situación actual. El análisis cualitativo intenta describir un movimiento en términos no numéricos. Los datos obtenidos de un análisis cualitativo pueden ser sustentados con un análisis cuantitativo y muchos proyectos de investigación son formulados de esta manera. La evaluación de un análisis cualitativo se basa en la habilidad del entrenador para reconocer los momentos críticos de la ejecución o del gesto deportivo; el análisis

cualitativo es el método predominante usado por los entrenadores 0 de los profesores de educación física en el análisis de los movimientos de sus deportistas. En la mayoría de los casos la observación visual es el procedimiento empleado, por medio de grabación de videos para el análisis cualitativo.

Pateo:

El pateo es una técnica fundamental y versátil usada para pasar, disparar y despejar. Los verdaderos aficionados al fútbol se refieren al disparo del balón porque suena más controlado.

\section{METODOLOGÍA:}

Esta propuesta de investigación se fundamenta en un diseño NO EXPERIMENTAL, de tipo descriptivo, para un caso, es decir es un estudio de caso, de un deportista que hace parte del equipo representativo de la UPTC de Tunja, que permitirá describir la técnica deportiva desde el objetivo de la biomecánica de analizar la ejecución del deportista, en términos de Aguado, la segunda de las tres clases de tareas de la Biomecánica Deportiva, los comentarios de tipo descriptivo se basan en los datos con un enfoque cuantitativo que se pueden tomar con el material descrito.

MATERIALES: cámara, programa Kinovea, Microsoft Excel.

Descripción del gesto técnico: pateo en el futbol sala.

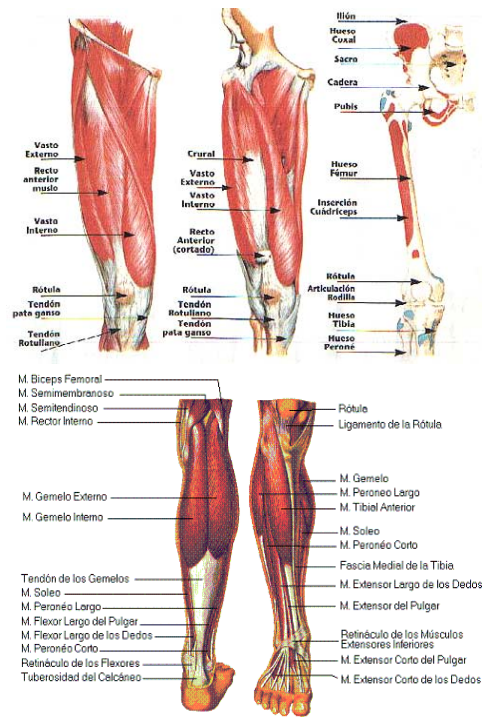

http://entrenadordefutbol.blogia.com/temas/musculos.php

Entre los músculos que intervienen en el golpeo, Weineck (1995), destaca la acción del cuadriceps de la pierna que ejecuta el golpeo, así como la acción de flexión de los músculos psoas iliaco, tensor de la fascia lata, recto femoral, y la contracción de los 
músculos de la pared abdominal. Respecto a la pierna de apoyo, destaca la acción que realizan el glúteo mayor, los isquiotibiales, el cuadriceps y el tríceps sural. Por otro lado se ha determinado que la función que desempeñan todos los músculos (flexores y extensores de cadera y flexores $y$ extensores de rodilla) es en alguna fase del golpeo agonista y en otra se convierte en antagonista, además se ha advertido sobre la importancia de la activación de los flexores de la rodilla para la prevención de la hiperextensión y posible daño en la rodilla en el golpeo (De Proft y cols, 1988; Luhtanen, 1999).

También se ha puesto de manifiesto un incremento en la eficacia de la musculatura extensora de la rodilla a nivel muscular a la hora de contraerse para el golpeo, debido a un pre-estiramiento mayor de los extensores de la rodilla y un tiempo de acoplamiento menor entre las fases excéntrica y concéntrica (Gutiérrez y Soto, 1992).
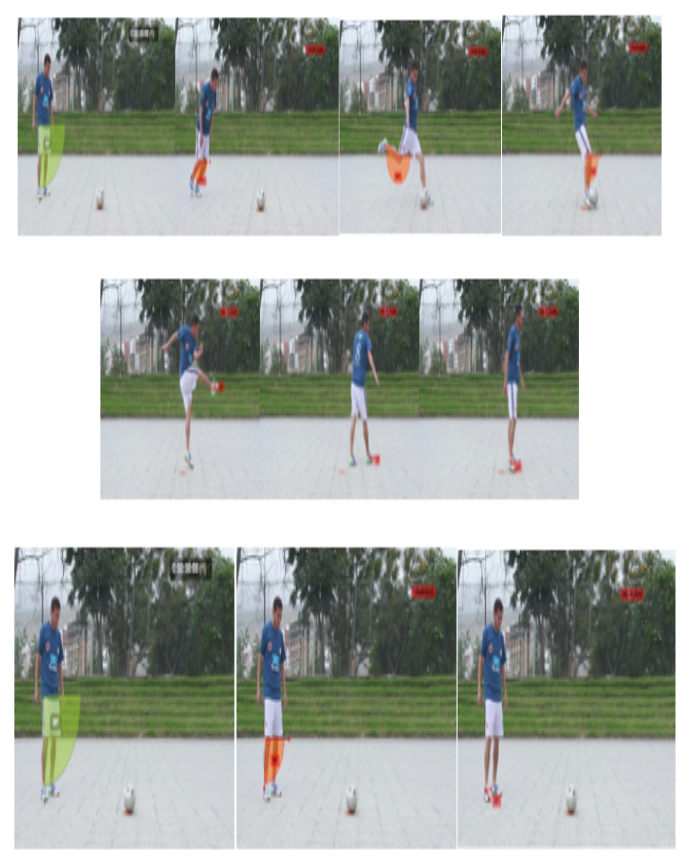

Fase inicial

El miembro inferior derecho está dedicado a la carga y la de ambulación, las cuales alternativamente nos proporcionan lo que conocemos como carrera en un jugador de fútbol sala que está en constante movimiento, éste no solo va a ampliar su base de sustentación, sino que un factor aún más importante es el centro de masa en el cuerpo. En esta fase es conveniente estar atentos a tres aspectos que intervendrán en el gesto. Son la carrera hacia el balón, la posición del pie de apoyo y la pierna de apoyo.

El cuádriceps se contrae para mantener estable la rodilla, puesto que en condiciones normales esta no es recta, durante esta fase del desplazamiento.

El peso queda encargado sobre cada una de las caderas correspondientemente, el glúteo medio es el encargado de permitir la abducción.

El impulso luego que el pie se encuentra apoyado en el suelo, corresponde a la articulación metatarso falange del dedo gordo, y que esta realice una hiperextensión. En la articulación de la rodilla, los músculos gemelos, sóleo y plantar delgado son vitales para el impulso, elevando el calcáneo desde su parte posterior y de esta manera quedar apoyado solo en la cabeza de los metatarsianos, generando como tal el impulso.

\begin{tabular}{|c|c|c|}
\hline \multicolumn{3}{|c|}{ PALANCAS } \\
\hline TERMINO & CUERPO HUMANO & SIMBOLO \\
\hline FUERZA & $\begin{array}{c}\text { MUSCULO PUNTO DE } \\
\text { INSERCIÓN }\end{array}$ & $\mathrm{F}$ \\
\hline RESISTENCIA & PESO DEL SEGMENTO & $\mathrm{R}$ \\
\hline BRAZO DE FUERZA & $\begin{array}{c}\text { DISTANCIA ENTRE LA } \\
\text { ARTICULACIONY LA INSERCIÓN }\end{array}$ & $\mathrm{BF}$ \\
\hline BRAZO DE & $\begin{array}{c}\text { DISNTACIA ENTRE EL PUNTO DE } \\
\text { APOYO(ARICULACIÓN) Y } \\
\text { CENTRO DE MASA DEL } \\
\text { SEGMENTO }\end{array}$ & BR \\
\hline
\end{tabular}

\section{CÁLCULOS BIOMECÁNICOS}

Musculo derecho

$\mathrm{Br}=40 \mathrm{~cm}^{*} 0,433=17.3 \mathrm{~cm}$
$\mathrm{P}=62 \mathrm{Kg}^{*} 9.8=607.6 \mathrm{~N}$
$\mathrm{PBrazo}=\left(607.6^{*} 9.63\right) / 100=58.5 \mathrm{~N}$
$\mathrm{~F}=(17.3 \times 58.5) / 6 \mathrm{~cm}=168.6 \mathrm{~N}$

Según Newton y Kraemer (Zapata, 2009), "la fuerza velocidad (fuerza explosiva según 
otros autores) es la responsable de la ejecución de actividades que requieren una secuencia de movimientos dirigida a producir una velocidad elevada de salida 0 de impacto en los cuerpos". La fuerza que realiza el músculo para vencer la resistencia y la velocidad de salida que se logra por la aceleración que se produce, es el factor que determinará la distancia que podrá recorrer el cuerpo, en este caso, el balón de futbol sala.

\begin{tabular}{|c|c|c|c|c|c|c|c|}
\hline \multicolumn{8}{|c|}{ CUADPOCWEEMTICONUSLOO } \\
\hline FOTOS & GRADOS & DSTANCA & DSACA & TEEPO(ses) & TIEACU & VEDT & 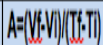 \\
\hline 1.44 & 4 & 1,3 & 1,3 & 0,48 & 0,43 & 3,0 & \\
\hline $14 \cdot 22$ & 3 & 1,0 & 2,3 & 0.27 & 0,70 & 3.6 & 2,2 \\
\hline 22.28 & 20 & 6,5 & 8,8 & 0.20 & 0,90 & 32,5 & 144,4 \\
\hline 28.35 & 46 & 149 & 237 & 0,23 & 1,13 & 650.0 & 141,2 \\
\hline 35.39 & 10 & 3,2 & 27,0 & 0,13 & 1,26 & 25,0 & $.307,5$ \\
\hline 3940 & 19 & 6.2 & 33,1 & 0,04 & 1,30 & 154,3 & 3232,4 \\
\hline 40.41 & 30 & 9,1 & 42.9 & 0,03 & 1,39 & 324,8 & 5864,1 \\
\hline 41442 & 20 & 65 & 494 & 0,03 & 1,30 & 2165 & 30090 \\
\hline 42,43 & $g$ & 2,9 & 52,3 & 0,04 & 1,40 & 73,1 & $.3560,4$ \\
\hline 43,44 & 14 & 4,5 & 50,8 & $0,0,3$ & 1,43 & 151,0 & 2616,5 \\
\hline 4446 & 18 & 5,8 & 02,7 & 0,07 & 1,50 & 80,5 & .972 .2 \\
\hline 4647 & 6 & 1,9 & 64,6 & $0,0,3$ & 1,53 & 65,0 & 608,7 \\
\hline 4756 & 64 & 20,8 & 85,4 & 0,30 & 1,83 & 69,3 & 14,4 \\
\hline 5682 & $g$ & 2,9 & 8893 & 0.87 & 2,70 & 3,4 & $.75,8$ \\
\hline $82: 100$ & $g$ & 29 & 91,3 & 0,00 & 330 & 49 & 2,5 \\
\hline
\end{tabular}

\begin{tabular}{|c|c|c|}
\hline $\begin{array}{c}\mathrm{T}= \\
\text { FUERZA }\end{array}$ & $\mathrm{P}=\mathrm{T} / \mathrm{t}$ ISTANCIA & $\mathrm{Cal}=\mathrm{T} / 1 \mathrm{cal}$ \\
\hline $\mathrm{T}=168,6 \mathrm{~N}^{*} 91,3$ & $\mathrm{P}=1.84 \mathrm{~J} / 3,30 \mathrm{~s}$ & $\mathrm{Cal}=1.84 \mathrm{~J} / 4.186 \mathrm{~J}$ \\
\hline $\mathrm{T}=1.84 \mathrm{~J}$ & $\mathrm{P}=0.55 \mathrm{~V}$ & $\mathrm{Cal}=0.43$ \\
\hline
\end{tabular}

Graficas del comportamiento del muslo durante la ejecución del movimiento.

Calculo biomecánico pierna.

\begin{tabular}{|l|}
\hline $\mathrm{Br}=37 \mathrm{~cm}^{*} 0,433=17.3 \mathrm{~cm}$ \\
$\mathrm{P}=62 \mathrm{Kg}^{*} 9.8=607.6 \mathrm{~N}$ \\
$\mathrm{P}-\mathrm{Braz0}=(607.6 * 4.53) / 100=27.5 \mathrm{~N}$ \\
$\mathrm{~F}=(17.3 \times 27.5) / 5 \mathrm{~cm}=95.1 \mathrm{~N}$
\end{tabular}

Graficas del comportamiento de la pierna durante la ejecución del movimiento

\begin{tabular}{|c|c|c|c|c|c|c|c|}
\hline \multicolumn{8}{|c|}{ CUADRO CINEMATICO PIERNA } \\
\hline Fotos & GRADOS & DISTANCAA & DIS.ACU & TIEMPO(seg) & TIEEACU & $\mathrm{V}=0 / \mathrm{T}$ & 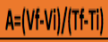 \\
\hline 1.14 & 17 & 5,5 & 5,5 & 0,43 & 0,43 & 12,8 & \\
\hline $14-22$ & 23 & 7,5 & 13,0 & 0,27 & 0,70 & 27,7 & 54,9 \\
\hline $22-28$ & 32 & 10,4 & 23,4 & 0,20 & 0,90 & 52,0 & 121,5 \\
\hline $28-35$ & 21 & 6,8 & 30,2 & 0,23 & 1,13 & 29,7 & $.97,0$ \\
\hline $35-39$ & 77 & 25,0 & 55,2 & 0,13 & 1,26 & 192,4 & 1251,8 \\
\hline $39-40$ & 13 & 4,2 & 59,4 & 0,04 & 1,30 & 105,6 & $.2170,6$ \\
\hline $40-41$ & 39 & 12,7 & 72,1 & 0,03 & 1,33 & 422,2 & 10556,2 \\
\hline 41-42 & 51 & 16,6 & 88,7 & 0,03 & 1,36 & 552,2 & 4330,8 \\
\hline $42-43$ & 40 & 13,0 & $.01,7$ & 0,04 & 1,40 & 324,8 & $.5684,1$ \\
\hline $43-44$ & 28 & 9,1 & 110,8 & 0,03 & 1,43 & 303,2 & $.721,8$ \\
\hline $44-46$ & 9 & 2,9 & 113,7 & 0,07 & 1,50 & 41,8 & $.3734,2$ \\
\hline $46-47$ & 5 & 1,6 & 115,3 & 0,03 & 1,53 & 54,1 & 412,5 \\
\hline 47.56 & 64 & 20,8 & 136,1 & 0,30 & 1,83 & 69,3 & 50,5 \\
\hline $56-82$ & 9 & 2,9 & 139,0 & 0,87 & 2,70 & 3,4 & $.75,8$ \\
\hline 82:100 & 22 & 7,1 & 146,2 & 0,60 & 3,30 & 11,9 & 14,2 \\
\hline
\end{tabular}

\begin{tabular}{|c|c|c|}
\hline TEFUERZA'DISTANCAA & $P=T / t$ & $C \mathrm{CA} \mid \mathrm{T} / \mathrm{Lat}$ \\
\hline$T=95,1 N^{4} 466,2$ & $P=13.9 . / 3,3005$ & Cal:13,9.9/4.1866] \\
\hline$T=13.91$ & PEA,2.2V & $(0 \mid=332$ \\
\hline
\end{tabular}

Graficas del comportamiento de la pierna durante la ejecución del movimiento.

Calculo biomecánico del pie.

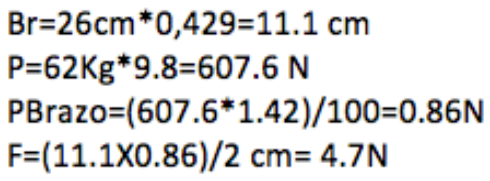

\begin{tabular}{|c|c|c|c|c|c|c|c|}
\hline \multicolumn{8}{|c|}{ CUADRO CINENATICO PIE } \\
\hline FOTOS & GRADOS & DISTANCIA & DIS.ACU & TIEMPO(seg) & TIE.ACU & $V=D / T$ & $A=(U V \cdot V i) /(T-T-T i)$ \\
\hline 1.14 & 2 & 0,6 & 0,6 & 0,43 & 0,43 & 1,5 & \\
\hline $14-22$ & 33 & 10,7 & 11,4 & 0,27 & 0,70 & 39,7 & 141,4 \\
\hline 2228 & 54 & 17,5 & 28,9 & 0,20 & 0,90 & 87,7 & 240,0 \\
\hline $28-35$ & 50 & 16,2 & 45,1 & 0,23 & 1,13 & 70,6 & $.74,3$ \\
\hline $35-39$ & 70 & 22,7 & 67,9 & 0,13 & 1,26 & 174,9 & 802,2 \\
\hline $39-40$ & 10 & 3,2 & 71,1 & 0,04 & 1,30 & 81,2 & $.2342,4$ \\
\hline 40-41! & 50 & 16,2 & 87,4 & 0,03 & 1,33 & $54,1,3$ & 15338,1 \\
\hline 41-42 & 46 & 14,9 & 102,3 & 0,03 & 1,36 & 498,0 & $-1443,6$ \\
\hline $42-43$ & 39 & 12,7 & 115,0 & 0,04 & 1,40 & 316,7 & $-4533,8$ \\
\hline 43-44 & 6 & 1,9 & 116,9 & 0,03 & 1,43 & 65,0 & $.8390,8$ \\
\hline 44-46 & 11 & 3,6 & 120,5 & 0,07 & 1,50 & 51,0 & $.198,9$ \\
\hline $46-47$ & 6 & 1,9 & 122,5 & 0,03 & 1,53 & 65,0 & 464,0 \\
\hline 47.56 & 8 & 2,6 & $.25,1$ & 0,30 & 1,83 & 8,7 & $.187,7$ \\
\hline $56-82$ & 12 & 3,9 & 128,9 & 0,87 & 2,70 & 4,5 & 4,8 \\
\hline $82: 100$ & 19 & 6,2 & 135,1 & 0,60 & 3,30 & 10,3 & 9,7 \\
\hline
\end{tabular}




\begin{tabular}{|c|c|c|}
\hline $\begin{array}{c}\mathrm{T}= \\
\text { FUERZA*DISTANCIA }\end{array}$ & $\mathrm{P}=\mathrm{T} / \mathrm{t}$ & $\mathrm{Cal}=\mathrm{T} / 1 \mathrm{cal}$ \\
\hline $\mathrm{T}=4.7 \mathrm{~N}^{*} 135,1$ & $\begin{array}{c}\mathrm{P}=634.9 \\
\mathrm{~J} / 3.30 \mathrm{~s}\end{array}$ & $\begin{array}{c}\mathrm{Cal}=634.9 \\
\mathrm{~J} / 4.186 \mathrm{~J}\end{array}$ \\
\hline $\mathrm{T}=634.9 \mathrm{~J}$ & $\mathrm{P}=192.3 \mathrm{~V}$ & $\mathrm{Cal}=151$ \\
\hline
\end{tabular}

Segunda fase
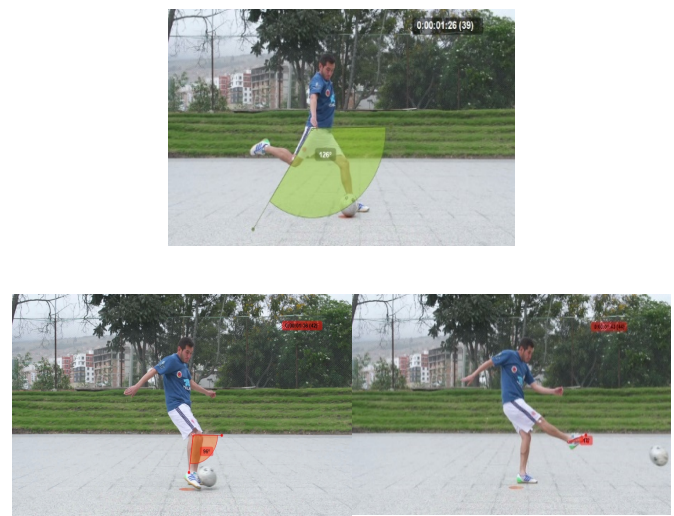

Una vez que la carrera o el desplazamiento hacia el balón es constante, el jugador mantiene una pierna en estado de apoyo mientras que la otra la mantendrá retrasada con respecto al tronco, de esta manera consigue mucho más impulso y fuerza para aplicar al balón.

La pierna de apoyo se mantiene en extensión completa, realizando una gran función los cuádriceps crurales, y el recto anterior colabora con la flexión de la cadera, la cual permite estabilizar la posición de la pierna.

A partir de esta posición, ahora, la pierna con la cual se ejecuta el movimiento, realiza una extensión de la rodilla principalmente, conjuntamente con la cadera, realizado por el cuádriceps en todas sus partes (vasto interno, externo, recto anterior y crural).

Adicionalmente los músculos posteriores de la pierna realizaran una contracción isométrica, para dar mayor estabilidad al movimiento.

\section{Tercera fase}

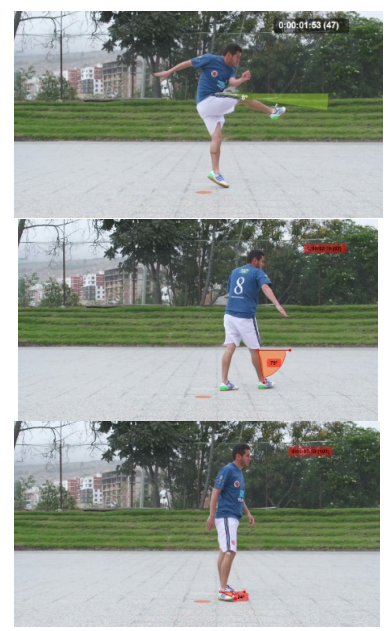

La pierna con la cual se realiza el golpe al balón se encuentra en extensión, mucho más adelantada con respecto al tronco, posterior se presenta una flexión de la pierna sobre la cadera, el pie con respecto a la pierna se encuentra en flexión plantar, lo cual permite presumir que aunque el mayor trabajo se presenta en la parte posterior de la pierna, la tibia también realiza una contracción isométrica de manera que se estabilice el movimiento y permita tener mayor eficacia en la ejecución del movimiento (pateo).

Todas y cada una de las anteriores fases son muy importantes. Muchos jugadores solo tienen en cuenta el momento de contacto. Pero es labor del entrenador darle importancia al resto porque pueden ser la clave para realizar de forma correcta un buen pateo.

\begin{tabular}{|c|c|}
\hline \multicolumn{2}{|c|}{ CENTROS DE GRAVEDAD } \\
\hline $\begin{array}{l}\text { FASES DEL MOVIMIENTO } \\
\text { (fotogramas) }\end{array}$ & CENTROS DE GRAVEDAD \\
\hline FASE DE PREPARACIÓN & $2,54-5,63$ \\
\hline FASE PRINCIPAL & $3,36-5,44$ \\
& $4,12-6,23$ \\
\hline & $2,71-5,28$ \\
FASE DE RECUPERACIÓN & $2,78-5,46$ \\
& $2,39-5,71$ \\
\hline
\end{tabular}




\section{Comportamiento del C.G}

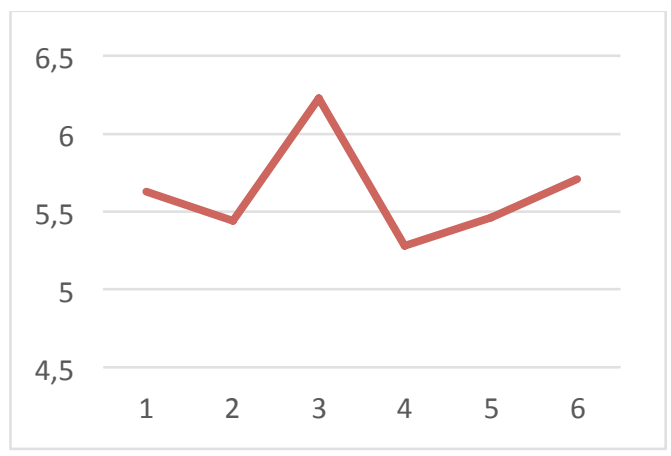

\section{CONCLUSIONES:}

Este análisis puede determinar en qué fase y cuál de los tres segmentos corporales debe ser intervenido ya sea en desarrollo de fuerza, o en flexibilidad si se evidencia movimientos insuficientemente amplios, también puede determinarse si el comportamiento del centro de gravedad es el conveniente según la lógica interna del movimiento, para el caso se observa un descenso del centro de gravedad en la preparación del movimiento, un ascenso en el momento del impacto, lo que va de acuerdo con lo esperado, así mismo se evidencia el mayor desarrollo de la velocidad en los fotogramas correspondientes al contacto (40-42) para los tres segmentos corporales implicados; sin embargo el mayor valor de velocidad de muslo y pie está en el intervalo 40-41 y en el muslo 41-42, lo que permitiría pensar en "mejorar" el movimiento del pie, que sea más coordinado con el de la pierna, ya que se da por hecho que la cadena cinemática tiene la secuencia en los pateos de muslo, pierna, pe y el dato encontrado podría significar que se hace la acción de movimiento del pie un poco antes de lo esperado para logra máxima eficiencia.

El mayor objetivo del análisis biomecánico es la observación detallada para poder hacer en forma práctica correcciones inmediatas, lo que permite inferir que este tipo de análisis elementales desde la cantidad de material utilizado, podría ser una importante herramienta de la cotidianidad del entrenador deportivo que pretende mejorar técnica.

\section{REFERENCIAS BIBLIOGRAFICAS}

Acero, José. (2010). Cinemática bidimensional y (2D) y biplanar (2P) del gesto deportivo. Cali.

Agudelo, C. (2012). Planificación del entrenamiento deportivo por Modelamiento. Editorial Kinesis. Armenia 2012

Barbero Álvarez José. Carlos. (1998). El entrenamiento de los deportes de equipo basado en estudios biomecánicos (análisis cinemático) y fisiológicos (frecuencia cardíaca) de la competición. Revista digital Efdeportes, Año 3. № 11. Buenos Aires. http://www.efdeportes.com/efd11a/biomec.ht $\mathrm{m}$

Donskoi, D.D, (1971). Biomecánica con fundamentos de la Técnica Deportiva. traducción de: SANTOS AMIGO, Mabel, editorial pueblo y educacion.1982 tomado de la edición en ruso de 1971.

Espinoza, Sanchez Matilde. (2005). Biomecánica Deportiva, disponible en: httpp://www.Sld.cu/galerías/pdf/sitios/rehabil itacion-equino/biomecanica.pdf. (fecha de acceso noviembre

de 2011).

García-Fojeda, Alberto; Biosca, Francesc; Vàlios, Joan Carles. (1997).La biomecánica: una herramienta para la evaluación de la técnica deportiva. Apunts. Educación Física y Deportes, 47:15-20.

Izquierdo Redin, Mikel (2008). Biomecánica y bases neuromusculares de la actividad física y el deporte. Madrid, España: Médica Panamericana. 
La enseñanza de los fundamentos técnicos del futbol y su incidencia en la participación en el campeonato escolar de la Escuela Benjamín Araujo de Patate en el año lectivo $2008-2009$.

Ramón Suárez, Gustavo (2006). Modelo biomecánico. Medellín, Colombia: VIREF Instituto de Educación Física, Universidad de Antioquia.

Ramón Suárez, Gustavo (2009). Biomecánica deportiva y control del entrenamiento. Medellín,

Colombia: Universidad de Antioquia, Instituto Universitario de Educación Física.

Zabala Díaz, Mikel; García Artero, Enrique; Lozano, Luis; Lozano Cid, Javier; Soto Hermoso,

Víctor M. (2006). Análisis de los golpeos de empeine y puntera en jugadores de élite de Futbol sala. Archivos de Medicina del Deporte, 23 (114): 274-282.

http://www.efisioterapia.net/articulos/analisis -biomecanico-del-golpeo-balon-futbol 